Document downloaded from:

http://hdl.handle.net/10251/64853

This paper must be cited as:

Herranz Herruzo, Jl.; Valero-Nogueira, A.; Martínez Giner, S.; Vila Jiménez, A. (2015). Untilted narrow-wall slots excited by parasitic dipoles in groove-gap waveguide technology. IEEE Transactions on Antennas and Propagation. 63(11):4759-4765. doi:10.1109/TAP.2015.2478144.

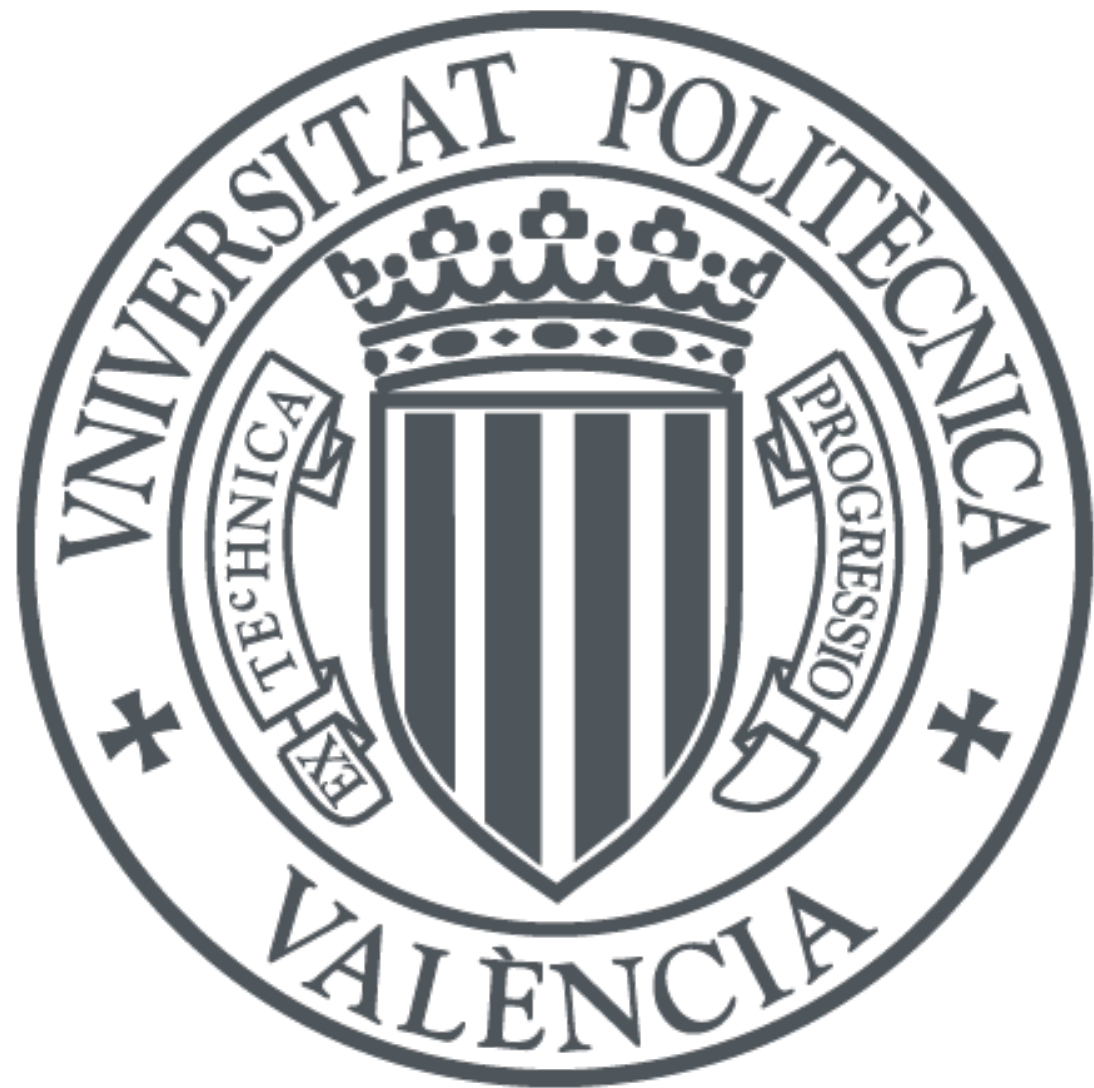

The final publication is available at

http://dx.doi.org/10.1109/TAP.2015.2478144

Copyright Institute of Electrical and Electronics Engineers (IEEE)

Additional Information

(C) 2015 IEEE. Personal use of this material is permitted. Permission from IEEE must be obtained for all other uses, in any current or future media, including reprinting/republishing this material for advertising or promotional purposes, creating new collective works, for resale or redistribution to servers or lists, or reuse of any copyrighted component of this work in other works. 


\title{
Untilted Narrow-Wall Slots excited by Parasitic Dipoles in Groove-Gap Waveguide Technology
}

\author{
José Ignacio Herranz Herruzo, Alejandro Valero-Nogueira, Sara Martínez Giner, Antonio Vila Jiménez
}

\begin{abstract}
This work describes a new way to excite untilted slots in the narrow wall of a rectangular waveguide. The radiating structure consists of an inclined parasitic dipole located within the waveguide, parallel to narrow wall and close to the untilted slot. Furthermore, the rectangular waveguide is advantageously replaced by a groove gap Waveguide so that nonwraparound resonant slots can be used. The concept is discussed in detail and demonstrated experimentally through a linear array.
\end{abstract}

Keywords-Slot arrays, waveguide arrays, millimeter-wave antenna arrays.

\section{INTRODUCTION}

Slotted waveguide array antennas have been widely used in radar and communication systems due to their ease of fabrication, agile aperture distribution control and low loss. The radiating element is a narrow slot cut either in the broad or in the narrow wall of a rectangular waveguide. When the slot is in the narrow wall, it must be inclined to excite it. However, slot inclination produces undesired cross-polarization. For the purpose of minimizing the presence of undesired cross-polarization, some alternatives were proposed in the past. In [1] Hashemi-Yeganeh and Elliott analyzed a structure formed by a pair of transversely placed (perpendicular to the waveguide axis) tilted wires that straddle the untilted slot and connected to both broad and side walls. Later, Hirokawa and Kildal [2] proposed a transverse conducting strip etched on both sides of a dielectric substrate to excite the untilted slot. However both procedures can be difficult to implement in practice and a later slot array design would be subjected to numerous influencing parameters. More recently Dogan and Civi [3] proposed a simpler alternative to excite untilted slots through the introduction of two small insets milled within the waveguide at opposite sides of the edge slot.

In [4], an alternative way to excite untilted slots in the narrow-wall of a waveguide was proposed. This new radiating structure basically consists of an untilted slot excited through the perturbation produced by a tilted parasitic dipole. This dipole is located within the waveguide, parallel to the narrow wall and at a given distance from the radiating slot. The tilting angle of the dipole would be responsible for the amount of power coupled to the slot. Up to $50 \%$ of the incident power can be radiated with this procedure.

Interestingly, as shown in [4], this new technique for exciting untilted slots reveals all its strength when combined with

This work was supported in part by the Spanish Ministerio de Economía y Competitividad under project TEC2013-47360-C3-3P.

The authors are with iTeam Institute, Universitat Politecnica de Valencia, 46022 Valencia, Spain (e-mail: avalero@dcom.upv.es). groove gap waveguide (GGW) technology [5]. Since GGW is a non contact metal waveguide it can greatly simplify fabrication of narrow-wall slotted waveguide arrays. Groove gap waveguide can be machined in two parts. One incorporates the waveguiding groove while the other is merely a cover, holding the radiating slots obtained out of a copper-clad laminate, having the slots etched on one side and the excitation dipoles on the other. This radiating layer would simply be placed on top of the groove and the nails without worrying about assuring electrical contact between parts.

In this paper the main features of this radiating element will be discussed. Particular attention will be devoted to mutual coupling effects since coupling, both external and internal, was found to be stronger than usual, given the slot spacing chosen for the array $(d=0.68 \lambda)$.

In section II the new radiating structure will be described in the context of a rectangular waveguide. The convenience of using a groove gap waveguide will also be discussed. In section III, the mutual coupling effect between radiating elements will be analyzed and a procedure to take it into account described. Finally, in section IV, a prototype array will be designed, fabricated and measured.

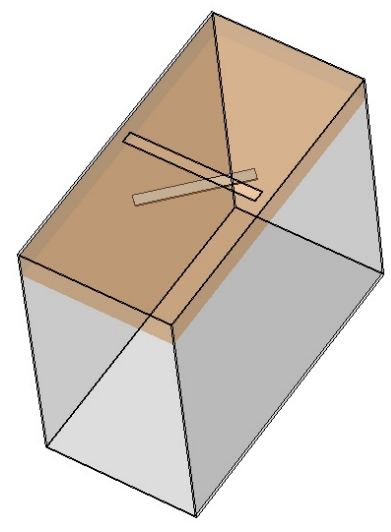

Fig. 1. Untilted slot in narrow-wall of rectangular waveguide, coupled to inclined parasitic dipole etched on dielectric substrate.

\section{Untilted Slot EXcited by a PARAsitic Dipole}

The radiating element consists of an untilted slot in the narrow-wall of the rectangular waveguide on its non-radiating position, excited by a parasitic dipole located parallel to the narrow wall and at a given distance from the slot, Fig. 1. The tilting angle of the dipole is the responsible for the slot excitation and the amount of power radiated. Furthermore, the 
dipole is etched on a substrate layer to simplify the fabrication process.

For the following parametric simulations, the working frequency is set to $37.5 \mathrm{GHz}$. The dimensions of the waveguide are $a \times b=7 \times 4 \mathrm{~mm}$, the slot length will be left fixed to $l_{\text {slot }}=3.5 \mathrm{~mm}$ and its width to $w_{\text {slot }}=0.3 \mathrm{~mm}$ (the same as dipole width). The variable parameters are the tilting angle of the dipole, $\alpha$, and its length, $l_{\text {res }}$. Actually, for each tilting angle, there is a dipole length which drives the slot-dipole element to resonance. $\alpha$ values are such that for $\alpha=0^{\circ}$, slot and dipole are parallel, while for $\alpha=90^{\circ}$ they are orthogonal.

In Fig. 2 the power radiated by the slot under resonance condition can be seen as a function of $\alpha$. Each $\alpha$ has an associated dipole length not shown here for brevity. The power radiated has been obtained analyzing the $S$-parameters from CST [6] and using the following equation $P=1-\left|S_{11}\right|^{2}-\left|S_{21}\right|^{2}$.

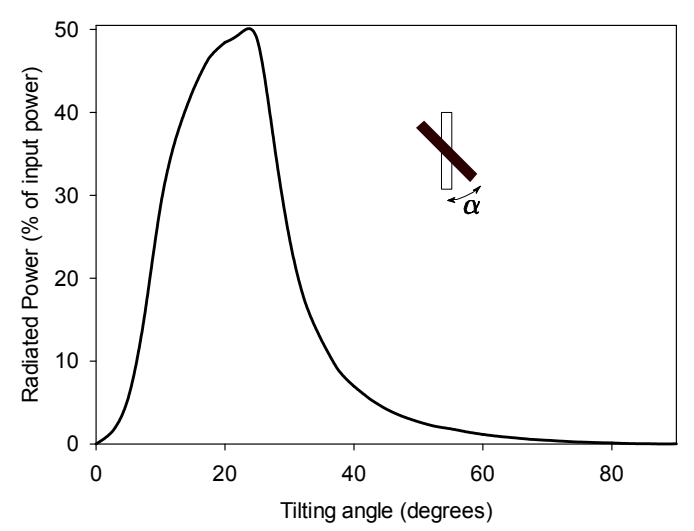

Fig. 2. Power radiated by the untilted slot in the narrow wall of a rectangular waveguide as a function of dipole tilting.

The so-defined radiating structure has two non-radiating situations based on dipole tilting angle. The first one is when the dipole is transverse to the waveguide propagation axis $\left(\alpha=0^{\circ}\right)$, and therefore exhibits the same orientation as the guided electric field. In that case the coupling with the incident mode is maximum but the dipole is parallel to the slot and cannot excite it. The second case is when the dipole is oriented along the propagation axis, $\left(\alpha=90^{\circ}\right)$. Now the dipole does not couple to the incident mode and can not excite the slot. In principle both slopes of the graph can be used to control amplitude tapering in an eventual array design. However both slopes are very steep, making array design quite sensitive to dipole tilting errors. The reason for such a narrowband behaviour is indeed the length of the slot which is far from resonance. One way to alleviate this problem is the use of wraparound slots as it is conventionally done for narrow wall arrays. The alternative considered in this work is to use newly proposed groove gap waveguides so that slots can extend beyond the waveguide limits and into the bed of nails surrounding the waveguiding groove.

\section{A. Groove Gap Waveguide Technology}

In order to better grasp the potential of this idea, a few words about the GGW concept are required. GGW Technology is based on wave propagation in the gap between two parallel surfaces providing a non-metal contact guiding structure [5]. This novel type of waveguide was developed to overcome some shielding challenges when dealing with rectangular waveguides in the millimeter-wave regime and above. Gap waveguides rely on the ideal cutoff property of the gap left between a perfect electric conductor (PEC) and a perfect magnetic conductor (PMC), provided that such gap spans no more than a quarter of a wavelength. In practice, a highimpedance surface plays the role of the PMC. This surface is made up of a bed of nails, one quarter of a wavelength in height at the center frequency. Under such conditions, when a properly sized groove is hollowed in the bed of nails, propagation can take place within it as if it was a conventional waveguide, but without resorting to shielding walls. Fig. 3 sketches the idea.

The GGW employed in this work should support an horizontally polarized $T E_{10}$ mode to play the role of an edgeslotted waveguide. Furthermore the gap and the upper plate can be conveniently accomplished with a copper-clad laminate, so that slots and parasitic dipoles are etched on each side. See Fig. 3a. As for the dimensions of the bed of nails, the interesting fact is that a wide range of values can provide the high-impedance condition required for the working band [7]. As a rule of thumb, nails must be around of a quarter of a wavelength in height with a periodicity ranging from $\lambda / 4$ to $\lambda / 2$. Nails themselves, as shown in Fig. $3 b$, can exhibit a variable thickness covering from 0.3 to 0.5 times the nail periodicity.

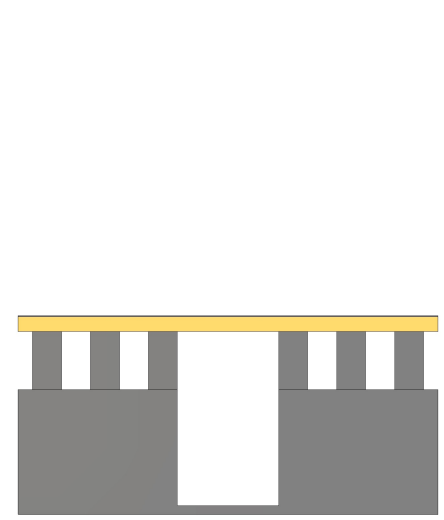

(a)

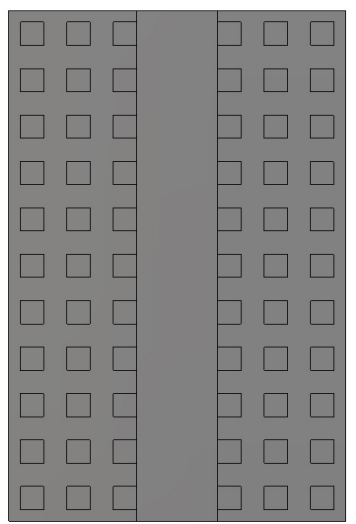

(b)
Fig. 3. (a) Cross-sectional view of horizontally polarized GGW with the gap filled with a dielectric material. Groove dimensions are $a \times b=5.88 \times 3.5$ $\mathrm{mm}$. (b) top view of the same GGW showing the surrounding bed of nails.

In the case considered here, the center frequency is 37.5 $\mathrm{GHz}$ and the dimensions of the groove have been chosen to be $a \times b=5.88 \times 3.5 \mathrm{~mm}$. Only fundamental $T E_{10}$ mode propagates at the working frequency range. Interestingly, Fig. 4 shows the propagation constants in GGW and rectangular waveguide for the same hollow dimensions. Nail periodicity 
could be easily adjusted to achieve virtually the same propagation constant at the band of interest. This result shows how similar both types of guides can be made in practice and suggests that some results can be readily extrapolated. Other dimensions used in the GGW are nails height, width and periodicity, which are 2,1 and $2 \mathrm{~mm}$, respectively. Substrate used is $0.508 \mathrm{~mm}$ in thickness, with a permittivity of $\varepsilon_{r}=2.2$.

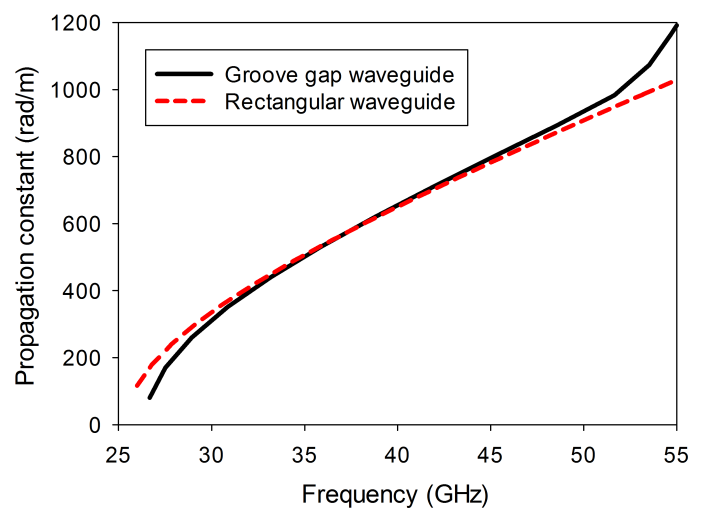

Fig. 4. Comparison between propagation constants in GGW and rectangular waveguide for the same hollow dimensions.

\section{B. Untilted slot in Groove Gap Waveguide}

Let us evaluate now the slot-dipole element in a GGW. Since the upper lid lacks any sidewalls, slots can be made longer. In our case, slot length is set to $4.1 \mathrm{~mm}$. This value is close to the resonant length and can lead slot-dipole element to resonance for a wide range of dipole tiltings. In Fig. 5a the power radiated by this slot under resonance condition is shown as a function of dipole tilting angle. It can be observed that the down slope is smoother than in Fig. 2, making the element less sensitive to fabrication tolerances. Corresponding dipole length is shown below.

As can be seen, slot-dipole element exhibits three parameters to control weighting and resonance: slot length and dipole length and tilting. However, in practice slot length can be left fixed, which can be interesting when dealing with external mutual coupling in an array. Dipole tilting provides control over slot weight, while resonance can be achieved adjusting dipole length. Slot-dipole element behaves like a shunt admittance. This assertion can be readily verified by checking whether the element complies with the condition $S_{21}=1+S_{11}$ or not. Table I shows to what extent this condition is assessed for two meaningful cases. One exhibits a strong coupling $\left(\alpha=25^{\circ}\right)$ and the other a weak one $\left(\alpha=80^{\circ}\right)$. Values are given as $S_{21}-S_{11}$ for table compactness. The shift observed with respect to unity is acceptable for design purposes and it is in the order of one would achieve for a conventional shunt slot in the broad wall of a rectangular waveguide, for equivalent coupling levels.

$S$-parameters were computed numerically using the model in Fig. 6. Nevertheless, a numerical TRL calibration was required to dembed $S$-parameters since a direct computation

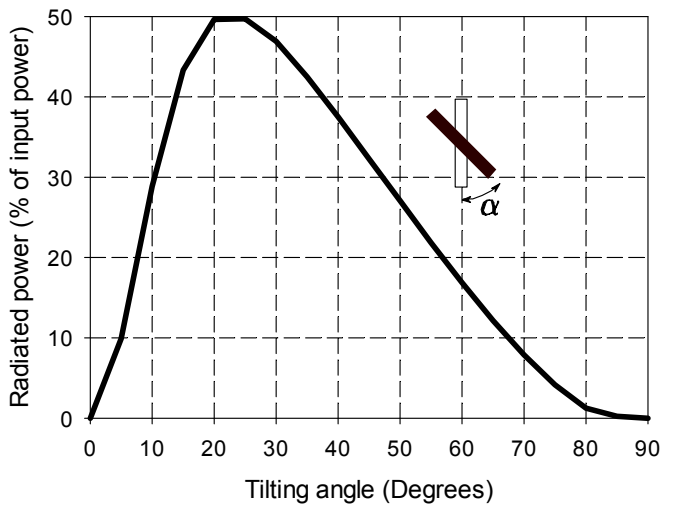

(a)

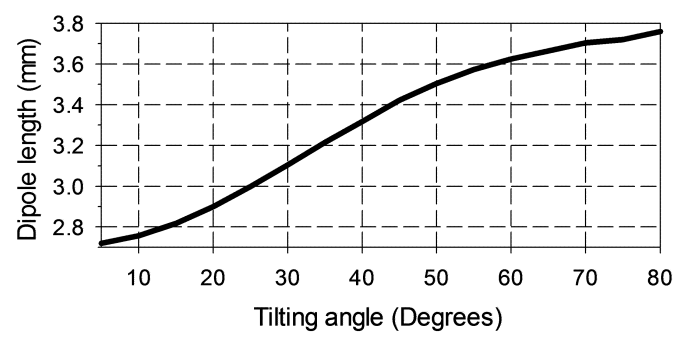

(b)

Fig. 5. (a) Power radiated by the untilted slot in the narrow wall of a GGW as a function of dipole tilting. (b) Dipole lengths driving slot-dipole element to resonance for each dipole tilting.

TABLE I. SHUNT CONDITION CHECKOUT

\begin{tabular}{c|c|c}
\hline \multirow{2}{*}{$\begin{array}{c}\text { Frequency } \\
(\mathrm{GHz})\end{array}$} & \multicolumn{2}{|c}{$S_{21}-S_{11}$} \\
\cline { 2 - 3 } & $\alpha=25^{\circ}$ & $\alpha=80^{\circ}$ \\
\hline 37.00 & $1.0005+0.0083 j$ & $1.0006+0.0113 j$ \\
37.25 & $1.0005+0.0054 j$ & $1.0006+0.0107 j$ \\
37.50 & $1.0003+0.0075 j$ & $1.0006+0.0103 j$ \\
37.75 & $1.0003+0.0071 j$ & $1.0006+0.0098 j$ \\
38.00 & $1.0003+0.0069 j$ & $1.0006+0.0094 j$ \\
\hline
\end{tabular}

would have led to significant errors in weakly coupled elements, due to the discontinuity between the GGW and the waveguide input ports.

Therefore, as it is common practice, an equivalent-network representation can be obtained from $S_{11}$-parameter through

$$
\bar{Y}_{\text {shunt }}=\frac{-2 S_{11}}{1+S_{11}}
$$

The characterization of the radiating structure is focused on finding the dipole length, $l_{\text {res }}$, that makes the slot resonant for each dipole tilting, $\alpha$. The slot length, $l_{\text {slot }}$, is left fixed for convenience at $4.1 \mathrm{~mm}$, and its resonance appears when the admittance imaginary part crosses zero. As can be seen in Fig. 7, for a given $\alpha=65^{\circ}$, resonance is achieved for $l_{\text {res }}=3.65$ $\mathrm{mm}$. A less coupled slot $\left(\alpha=80^{\circ}\right)$ is also shown for the sake of comparison. Notice that resonance lengths are in line with values shown in Fig. $5 b$. 


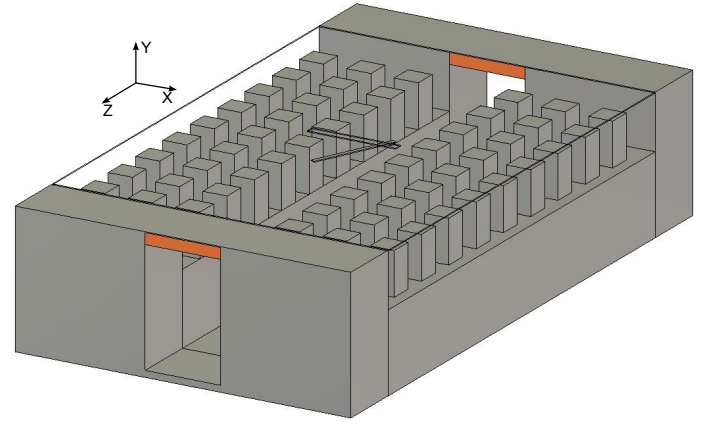

Fig. 6. Groove gap waveguide with transitions to rectangular waveguide

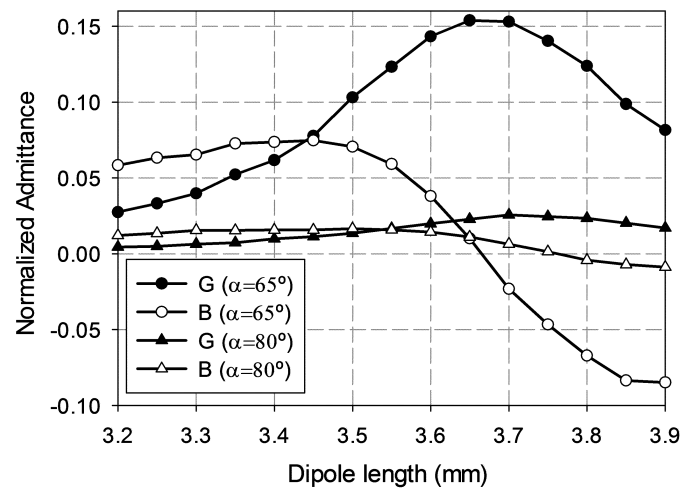

Fig. 7. $\bar{Y}_{s}$ for the slot-dipole element being $l_{\text {slot }}=4.1 \mathrm{~mm}$.

\section{Mutual Coupling ANALysis}

For demonstrating the feasibility of the slot-dipole element, an actual waveguide array should be attainable. A linear array configured using this type of element will exhibit important external coupling among slots since they are aligned along their $\mathrm{E}$ plane. This effect can even be aggravated in those cases where array spacing is chosen close to $\lambda / 2$, as internal coupling will play a noticeable role too. In the case considered here, both types of coupling effects will be present. The technique followed here to compensate for the coupling effects was long ago proposed by Elliott for resonant arrays of shunts slots in rectangular waveguide [8]. A direct application of the expressions in [8] is not possible here, though, since modes in GGWs are not those of rectangular waveguides, and are not known analytically, either. Therefore, some values obtained by Elliott from analytical expressions have had to be computed here numerically. In the following, some of the derivations in Elliott's procedure are reformulated with the only purpose of pointing out those calculations.

\section{A. Design equations}

As in [8] the starting point is an integral equation obtained enforcing continuity of tangential components of magnetic field in the slot

$$
\mathbf{H}_{\text {tan }}^{\text {ext }}(\mathbf{M})=\mathbf{H}_{\text {tan }}^{\text {scat }}(-\mathbf{M})+\mathbf{H}_{\text {tan }}^{\text {inc }}
$$

whose solution determines the unknown magnetic current M, i.e., the electric field in the slot. Superscripts ext, scat and inc stand for external, internally scattered and incident mode, respectively. Choosing sets of basis and weighting functions, $\left\{\mathbf{m}_{n}\right\}$ and $\left\{\mathbf{w}_{n}\right\}$ respectively, the operator above can be expressed in matrix form as

$$
[Y][V]=[I]
$$

where $[Y]=\left[\left\langle\mathbf{w}_{m}, \mathbf{H}_{\text {tan }}^{\text {ext }}\left(\mathbf{m}_{n}\right)+\mathbf{H}_{\text {tan }}^{\text {scat }}\left(\mathbf{m}_{n}\right)\right\rangle\right]_{N \times N}$ is the admittance matrix and $[I]=\left[\left\langle\mathbf{w}_{m}, \mathbf{H}_{t a n}^{i n c}\right\rangle,\right]_{N \times 1}$ the excitation vector.

As it is explained in [9], a relation between the admittance of a single slot in an infinite waveguide and its corresponding $S_{11}$-parameter can be written as

$$
S_{11}=-[I]^{t}[Y]^{-1}[I]
$$

where the superscript $t$ means transpose.

Without loss of generality, in the following, only one basis function will be assumed to simplify notation. Therefore, it is imposed that $\mathbf{m}=\mathbf{w}=\frac{1}{w_{s}} \cos \left(\frac{\pi}{l_{s l o t}} x^{\prime}\right) \mathbf{x}$, being $w_{s}$ the slot width. Then, reflection coefficient turns out to be $S_{11}=$ $-I^{2} / Y$

Introducing the simplified version of (4) in (1), a explicit relation between the equivalent shunt admittance and the slot admittance for a single slot can be formulated

$$
\bar{Y}_{\text {shunt }}=\frac{2 I^{2}}{Y-I^{2}}
$$

Given an array of $\mathrm{N}$ slots, the active admittance of $n$-th slot, considering both external and internal mutual effects is expressed as

$$
Y_{n}^{\prime}=Y_{n}+Y_{n}^{\text {mutual }}=Y_{n}+\sum_{m \neq n} Y_{n m}^{e x t} \frac{V_{m}}{V_{n}}+\sum_{m \neq n} Y_{n m}^{\text {int }} \frac{V_{m}}{V_{n}}
$$

where $Y_{n m}^{i n t}$ here only accounts for coupling due to internally scattered evanescent modes, since fundamental mode coupling is implicit in the equivalent network model. Now, Eq. (5) is valid also for active admittances, therefore, for the $n$-th slot,

$$
\bar{Y}_{n, \text { shunt }}^{a}=\frac{2 I_{n}^{2}}{Y_{n}+Y_{n}^{\text {mutual }}-I_{n}^{2}}
$$

In (7), the slot self-admittance, $Y_{n}$, can be conveniently replaced by its expression in terms of $\bar{Y}_{\text {shunt }}$ given by (5), and after a few manipulations it is arrived at

$$
\frac{1}{\bar{Y}_{n, \text { shunt }}^{a}}=\frac{1}{\bar{Y}_{n, \text { shunt }}}+\left(\frac{2 I_{n}^{2}}{Y_{n}^{\text {mutual }}}\right)^{-1}
$$

which is the active equivalent admittance of $n$-th slot, and indeed it is Elliott's second design equation, equation (33) in [8], although here it has been rewritten in a more general 
fashion, amenable for any kind of waveguide supporting the slots. Notice that term $I_{n}$ is equivalent to $f_{n}$ in [8]. Evidently, $I_{n}$, the weighted incident magnetic field in $n$-th slot, will have to be computed numerically for GGWs as it is done in [9]. External coupling can be computed directly, using free-space Green's function, while internal coupling will be computed, as part of the iterative process, when required.

\section{B. Iterative procedure}

$\bar{Y}_{\text {shunt }}$ and $I$ values are computed numerically using CST Microwave Studio [6] and tabulated for a discrete set of dipole tiltings and lengths $\left(\alpha_{p}, l_{p}\right)$. Likewise, the external mutual coupling terms, relevant to each slot, can be calculated and stored in advance as well. Notice that the length of the slots remain unaltered throughout the optimization. Initially, internal mutual coupling will be neglected.

As starting guess for $Y_{n, \text { shunt }}^{a,(0)}$, the target voltage of the slots squared, $K\left(V_{n}^{s}\right)^{2}$, can be used. Superscript (0) anticipates the need for an iterative procedure to solve for the desired dipole parameters. In each iteration, a new table containing $\bar{Y}_{\text {shunt }}^{a,(i)}$ values is generated using (8). From it, a set of dipole parameters $\left(\alpha_{n}^{(i)}, l_{n}^{(i)}\right)$ for the desired $Y_{n, \text { shunt }}^{a}$ is extracted using a conventional 2D search algorithm. Iterating is required since every new set of dipole parameters implies different coupling. Therefore new active admittance values are calculated with the so-called Elliott's first design equation, eq. (10) in [8].

$$
\bar{Y}_{n, \text { shunt }}^{a(i+1)}=K I_{n}^{(i)} V_{n}^{s}
$$

The process above is repeated until convergence for the dipole parameters is achieved. For the sake of completeness, notice that admittance values in any iteration must satisfy the matching condition $\sum \bar{Y}_{n, \text { shunt }}^{a,(i)}=1$, assuming an end-fed array. $K$ in (9) is calculated to comply with such condition.

When slot spacing is less than $0.7 \lambda$, internal coupling effects should not be neglected, otherwise the process above would not lead to the expected solution. Fortunately, these effects can be estimated and included in the analysis. For that, actual values of slot voltages, $V_{n}^{s}$, and the array input reflection coefficient, $\rho_{i n}$, must be computed numerically for the array simulated with the resulting set $\left(\alpha_{n}^{(P)}, l_{n}^{(P)}\right)$ calculated in previous stage after $P$ iterations. Obviously, this is only possible for small arrays. Then, active admittances are computed using (9) with actual $V_{n}^{s}$ values and forcing them now to comply with the matching condition $\sum \bar{Y}_{n, \text { shunt }}^{a}=\bar{Y}_{i n}$, being $\bar{Y}_{i n}=\left(1-\rho_{i n}\right) /\left(1+\rho_{i n}\right)$. With these values, (6)-(8) can be solved for internal contribution of mutual coupling, as external mutual coupling is known. From here the procedure proceeds in the iterative way above, but including internal coupling in $Y_{n}^{\text {mutual }}$.

\section{ApPliCATION TO SLOTTED GGW ARRAY DESIGN}

\section{A. Taylor distribution array}

The procedure above has been used to design an end-fed eight-element standing-wave series array with Taylor distribution and a sidelobe target of $-20 \mathrm{~dB}$. The GGW dimensions are those used in section II-A. Computed waveguide wavelength is $\lambda_{g}=10.9 \mathrm{~mm}$, therefore slot spacing at 37.5 $\mathrm{GHz}$ is $d=\lambda_{g} / 2=0.68 \lambda$, implying that internal coupling compensation was required. Table II shows the result of the optimization. A comparison between targeted and achieved voltages, $V_{\text {target }}^{s}$ and $V_{\text {achieved }}^{s}$, respectively, can be observed. Next to it, the dipole parameters are shown. A first set of dipole parameters are displayed under column $Y^{\text {ext }}$ for the first stage of the algorithm, when internal coupling is neglected. To the right, the final set of parameters are shown under column named $Y^{e x t}+Y^{i n t}$, emphasizing that both external and internal coupling were compensated in the optimization. Achieved voltages are for these set of parameters.

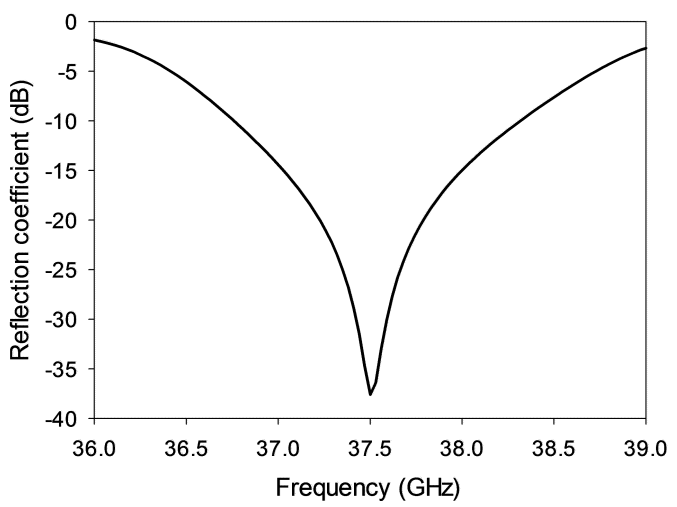

Fig. 8. Simulated reflection parameter $S_{11}$ for the end-fed 8-element array.

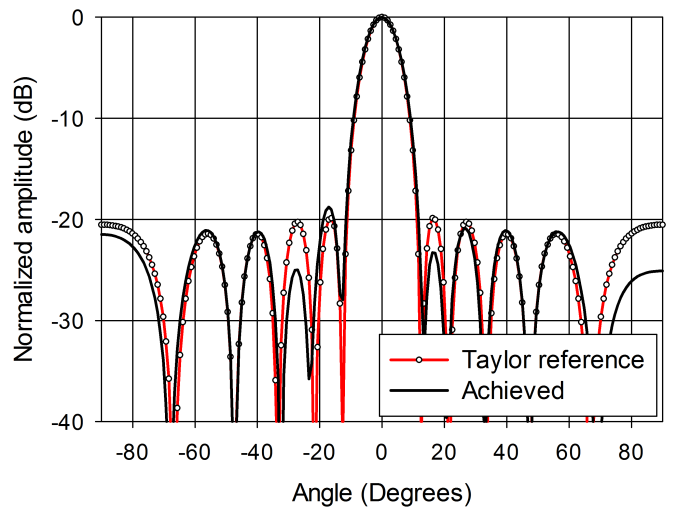

Fig. 9. Simulated E-plane radiation pattern for the end-fed 8-element array. Targeted Taylor pattern is shown as well for reference.

It is worth noticing that even though dipole parameters exhibit a symmetrical pattern with respect to the array center, achieved voltages do not. The explanation for such unexpected result comes from within the GGW. Indeed, unlike conventional waveguides, which exhibit an invariant cross-section along the propagation axis, GGWs feature a nail periodicity so that each slot actually "sees" a different nail environment. Relative position of the slot with respect to the nails differs slightly, in general, from that in the single slot model and has an impact on actual shunt admittance. No doubt, this feature 
TABLE II. VOltages AND DiPOLE PARAMETERS ACHIEVED FOR 8-ELEMENT TAYLOR ARRAY

\begin{tabular}{cc|ccc|cc}
\hline \multicolumn{2}{c}{ Slot voltages } & \multicolumn{2}{c}{$Y^{\text {ext }}$} & \multicolumn{2}{c}{$Y^{\text {ext }}+Y^{\text {int }}$} \\
\hline$V_{\text {target }}^{s}$ & \multicolumn{2}{c|}{$V_{\text {achieved }}^{s}$} & $\alpha_{n}^{\circ}$ & $l_{n}(\mathrm{~mm})$ & $\alpha_{n}^{\circ}$ & $l_{n} \mathrm{~mm}$ \\
\hline 0.55 & 0.48 & $-10^{0}$ & 74.2 & 3.74 & 74.3 & 3.85 \\
0.68 & 0.62 & $-8.3^{0}$ & 79 & 3.79 & 76.6 & 3.82 \\
0.86 & 0.89 & $-1.8^{0}$ & 72.8 & 3.77 & 67.8 & 3.79 \\
1 & 1.00 & $0^{0}$ & 69 & 3.73 & 66 & 3.79 \\
1 & 0.96 & $-3.6^{0}$ & 69 & 3.73 & 66 & 3.79 \\
0.86 & 0.87 & $-5.3^{0}$ & 72.8 & 3.77 & 67.8 & 3.79 \\
0.68 & 0.65 & $-3.4^{0}$ & 79 & 3.79 & 76.6 & 3.82 \\
0.55 & 0.51 & $-5.6^{0}$ & 74.2 & 3.74 & 74.3 & 3.85 \\
\hline
\end{tabular}

is impairing the algorithm convergence too. Still, a reasonably good outcome is observed both in reflection coefficient and radiation pattern, as Figs. 8 and 9 show. First sidelobe on the left is $-18.85 \mathrm{~dB}$, around $1 \mathrm{~dB}$ above desired level.

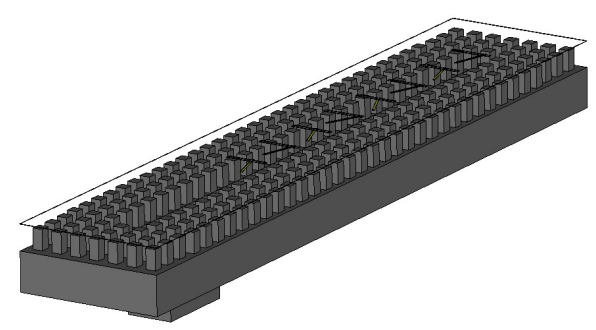

(a)

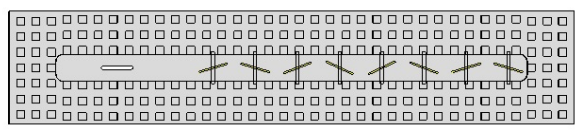

(b)

Fig. 10. (a) Perspective and (b) Top view of the complete 8-element Taylor slot-array antenna.

\section{B. Array feed}

From a practical point of view sometimes it is preferable feeding the array from the back. In this subsection a simple slot transition is described which will be later used in the fabricated prototype. A longitudinal slot couples the slotted GGW array to a conventional coaxial-to-waveguide adapter as depicted in Fig. 11. An optimization is performed on the coupling slot distance to backshort, $L$, and on coupling slot length and width, $h_{i}$, and $w_{i}$, respectively.

The coaxial-to-waveguide adapter has standard waveguide cross-section dimensions $h_{a} \times w_{a}=7.11 \times 3.56 \mathrm{~mm}$. Notice that the waveguide to coaxial transition is not considered in the model for optimization. A waveguide port is used instead. Also, a waveguide port is used as output in the GGW. The transition is optimized as a two-port network. Values achieved after optimization are: $L=7.335 \mathrm{~mm}, h_{i}=4.2 \mathrm{~mm}$ and $w_{i}=0.5 \mathrm{~mm}$.
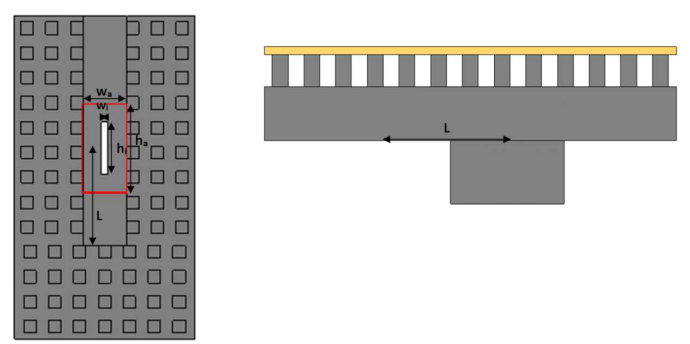

Fig. 11. Top and side view of the feeding transition.

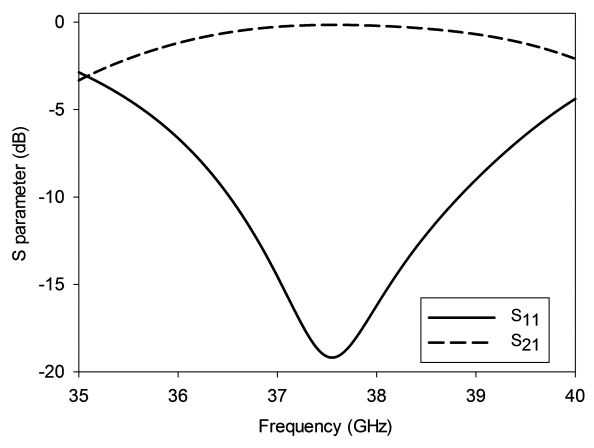

Fig. 12. S-parameters obtained for the feeding transition.

In Fig. 12 the S-parameters of this two-port network are shown. The whole antenna is shown in Fig. 10.

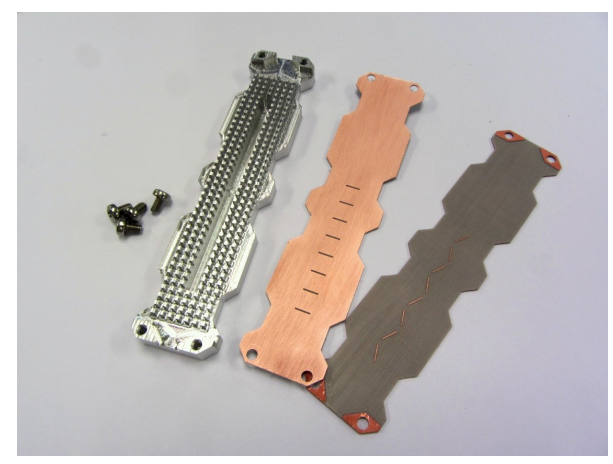

Fig. 13. Manufactured prototype of the 8-element Taylor slot-array antenna. Groove waveguide is on the left side. Radiating layer showing both faces is on the right.

\section{Fabrication and Measurement Results}

A prototype was fabricated according to the model shown in Fig. 10. In Fig. 13 a picture of the actual prototype is shown. As can be seen, it can be fabricated in two independent parts and put together, being the alignment between them the only concern to take care of.

Fig. 14 shows the measured $S_{11}$ coefficient well-tuned at the design frequency of $37.5 \mathrm{GHz}$. In Fig. 15 the measured radiation pattern at the center frequency is compared with the pattern achieved during design. Only a slight degradation 
in sidelobes is observed. Sidelobe is increased in $0.4 \mathrm{~dB}$ with respect to design. In any case the resemblance between simulation an measurement is remarkable, suggesting that, contrary to what one may think, the technology proposed is not particularly sensitive to tolerance errors.

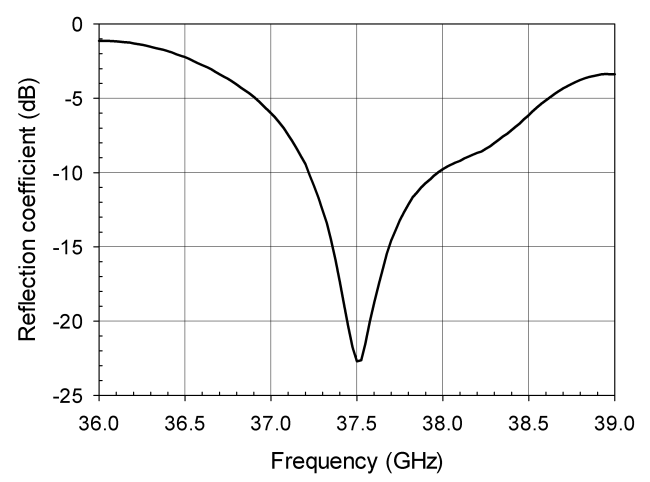

Fig. 14. Measured $S_{11}$ for the 8-element Taylor slot-array antenna.

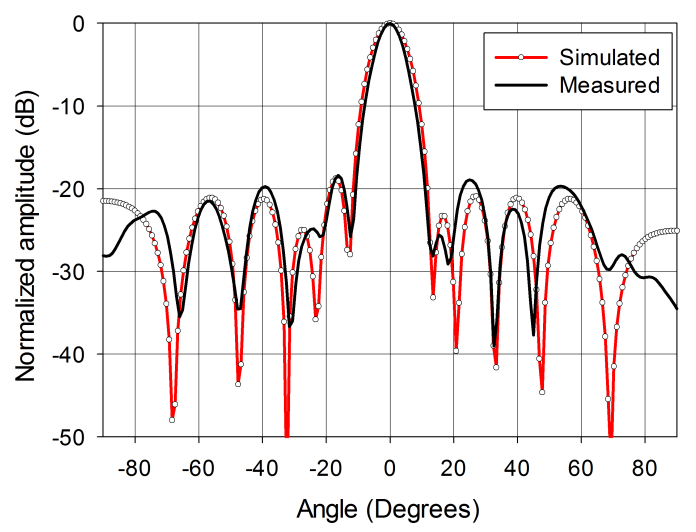

Fig. 15. Measured E-plane pattern compared with pattern achieved during design process.

Finally, for completing the radiation data, E-plane patterns at three frequencies are shown in Fig. 16. H-plane pattern is not shown since it is not considered relevant in this case.

\section{COnclusions}

The untilted narrow-wall slot in groove gap Waveguide technology excited using a parasitic dipole has been analyzed and succesfully proved experimentally in a slotted waveguide array. Strong coupling effects between elements had to be dealt with combining numerical observables and rigorous analysis.

\section{ACKNOWLEDGMENT}

The authors wish to thank the reviewers for their valuable suggestions that greatly contributed to improve the paper.

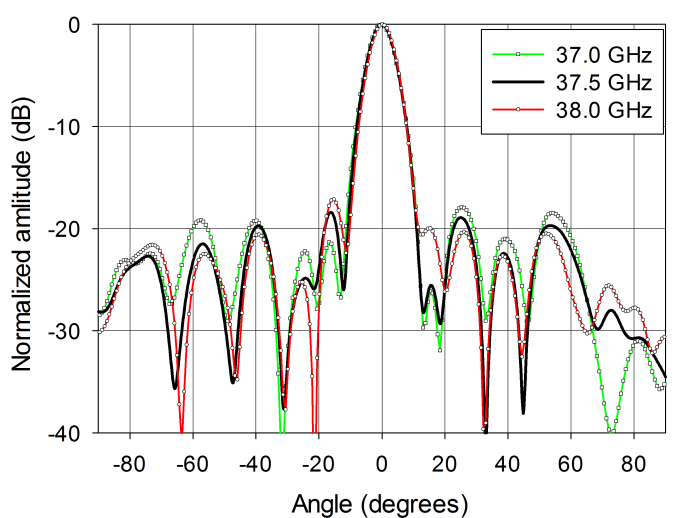

Fig. 16. Measured E-plane pattern at three frequencies.

\section{REFERENCES}

[1] S. Hashemi-Yeganeh, R.S. Elliott, "Analysis of untilted edge slots excited by tilted wires," IEEE Trans. Antennas and Propag., vol.38, no. 11, pp. 1737-1745, Nov. 1990

[2] J. Hirokawa,P.-S. Kildal, "Excitation of an untilted narrow-wall slot in a rectangular waveguide by using etched strips on a dielectric plate," IEEE Trans. Antennas and Propag., vol. 45 , no. 6, pp. 1032-1037, Jun. 1997.

[3] D. Dogan, O.A. Civi, "Edge wall slotted waveguide antenna with low cross polarization," IEEE Antennas and Propag. Soc. Symp., July 2010.

[4] S. Martínez Giner, A. Valero-Nogueira,J. Herranz-Herruzo, M. BaqueroEscudero, "Excitation of Untilted Narrow-Wall Slot in Groove Gap Waveguide by Using a Parasitic Dipole," European Conf. on Antennas and Propag., 2013.

[5] E. Rajo-Iglesias, P.-S. Kildal, "Groove gap waveguide: A rectangular waveguide between contactless metal plates enabled by parallelplatecutoff," European Conf. on Antennas and Propag., 2010.

[6] CST Microwave studio 2013. www.cst.com

[7] E. Rajo-Iglesias, P.-S. Kildal, "Numerical studies of bandwidth of parallel-plate cut-off realised by a bed of nails, corrugations and mushroom-type electromagnetic bandgap for use in gap waveguides,", IET Microw. Antennas and Propag., vol 5. no. 3, pp. 282-289, 200

[8] R.S. Elliott, "An improved design procedure for small arrays of shunt slots," IEEE Trans. Antennas Propagat., vol. AP-31, pp. 48-53; Jan. 1983.

[9] A. Valero-Nogueira, J.I. Herranz-Herruzo, M. Baquero, R. Hernandez, V. Rodrigo, "Practical derivation of slot equivalent admittance in periodic waveguides ," IEEE Trans. Antennas and Propag., vol. 61, no. 4, pp. 2321-2324, Apr. 2013.

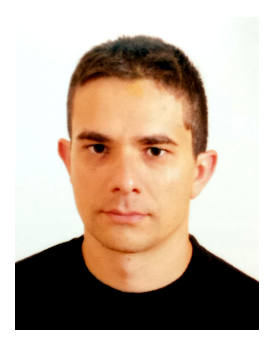

José Ignacio Herranz Herruzo was born in Valencia, Spain, in 1978. He received the Telecommunication Engineer degree from Universidad Politécnica de Valencia, Spain, in 2002. He is currently at his final stage of Ph.D. degree. He joined the Departamento de Comunicaciones at Universidad Politécnica de Valencia in 2002, where he is currently working as Assistant Professor. His main research interests include optimization of waveguide slot array antennas and efficient computational methods for planar structures. 
This is the author's version of an article that has been published in this journal. Changes were made to this version by the publisher prior to publication.

The final version of record is available at http://dx.doi.org/10.1109/TAP.2015.2478144

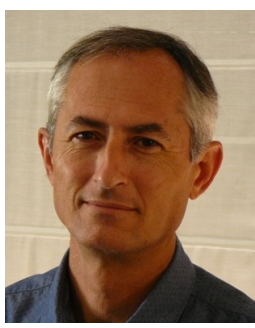

Alejandro Valero-Nogueira (S'92M'97-SM'09) was born in Madrid, Spain, on July 19, 1965. He received the Telecommunication Engineer degree from the Universidad Politécnica de Madrid, Madrid, in 1991 and the Ph.D. degree in Telecommunication from the Universidad Politécnica de Valencia, Valencia, Spain, in 1997. In 1992, he joined the Departamento de Comunicaciones, Universidad Politécnica de Valencia, where he is currently an Associate Professor. During 1999, he was on leave at the ElectroScience Laboratory, The Ohio State University, Columbus, where he was involved in fast solution methods in electromagnetics and conformal antenna arrays. His current research interests include computational electromagnetics, waveguide slot arrays, gap waveguides, the theory of characteristic modes and automated antenna design procedures.

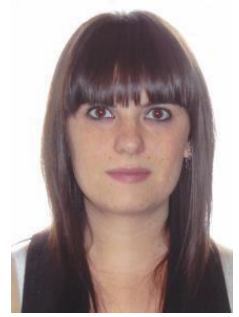

Sara Martínez Giner was born in Valencia, Spain, on September 3, 1990. She finished the Telecommunication Engineering studies in 2013, and the Master's Degree in Communication Technologies, Systems and Networks in 2014, both from the Universidad Politécnica de Valencia, Valencia, Spain. In February 2012, she started to research at the Electromagnetic Radiation Group, iTeam, at Universidad Politécnica de Valencia, characterizing and developing slotted waveguide arrays in Gap Waveguides. She is currently at Sistelbanda, Valencia, Spain, working as Validation Engineer for $4 \mathrm{G}$ femtocell netwoks.

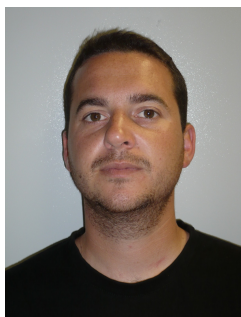

Antonio Vila Jiménez was born in Valencia, Spain, on June 18, 1981. He received the degree in Telecommunications Engineering, specialising in Telecommunications Systems from the Universidad Politécnica de Valencia, Spain, in 2007. He has been with the Instituto de Telecomunicaciones y Aplicaciones Multimedia (iTEAM) at the Universidad Politécnica de Valencia since 2007. His main research interests includes field antenna measurement and antenna fabrication. 